\title{
Estimation and Global Stability Analysis of PDAEs with Singular Time Derivative Matrix and Wetland Conservation Application
}

\author{
Yushan Jiang ${ }^{1,2}$ and Qingling Zhang ${ }^{1}$ \\ ${ }^{1}$ Institute of System Science, Northeastern University, Wenhua Road, Shenyang 110819, China \\ ${ }^{2}$ School of Mathematics and Statistics, Northeastern University at Qinhuangdao, Taishan Road, Qinhuangdao 066004, China \\ Correspondence should be addressed to Yushan Jiang; jys@neuq.edu.cn
}

Received 3 August 2015; Accepted 8 December 2015

Academic Editor: Carmen Coll

Copyright (c) 2016 Y. Jiang and Q. Zhang. This is an open access article distributed under the Creative Commons Attribution License, which permits unrestricted use, distribution, and reproduction in any medium, provided the original work is properly cited.

Some partial differential algebraic equations (PDAEs) system with singular time derivative matrices is analyzed. First, by PDE spectrum theory, this system is formulated as infinite-dimensional singular systems. Second, the state space and its properties of the system are built according to descriptor system theory. Third, the admissible property of the PDAEs is given via LMIs. Finally, the developed energy estimation method is proposed to investigate the global stability of PDAEs. The proposed approach is evaluated by an application in numerical simulations on some wetland conservation system with social behavior.

\section{Introduction}

Singular systems have abilities in representing dynamical systems in the areas of electrical circuits and multibody systems, chemical engineering and economic systems, and mechanical structure and biological systems. Singular systems are also referred to as descriptor systems, differential algebraic equations (DAEs) systems, implicit systems, or generalized state space systems [1]. A large number of fundamental ideas and results based on state space systems have been successfully extended to singular systems, such as controllability observability, pole assignment, stability, and stabilization [1-6]. In recent years, partial differential algebraic equations (PDAEs) have become an independent field of research. The applications and mathematical theory on PDAEs have attracted increasing attention to academics. The PDAEs research areas can be classified into three groups:

(i) Index analysis and solvability of PDAEs (see [7-13]).

(ii) Innovative and improved numerical methods to solve PDAEs (see [14-16]).

(iii) Control problems and optimization described by PDAEs (see [17-23]).
The structure analysis and solvability on PDAEs can be traced back to [24]. In [24], with the same method of line (MOL), there exist different spatial indexes between different numerical methods. In order to relate properties of the PDAEs to those of the resulting DAEs, it is necessary to have a concept of the index of possibly constrained PDAEs. Firstly, in [9], the perturbation index was defined on a class of liner time invariant PDAEs. On infinite domain, the wave solutions of PDAEs were also considered by [25]. In [26, 27], there was a systematic analysis about three different types of indexes (model, perturbation, and algebraic). In [28], a consistent representation of the solution of an initial boundary value problem for PDAEs was proposed. The index involved in the problem is characterized by means of Fourier and Laplace transformations. The differentiation index [29] of some general nonlinear PDAEs on hyper-plane domains is a generalization of the differentiation index of DAEs. It provides a way to determine Cauchy data on domain surface which must be consistent with the PDAEs. For the first time the perturbation index for a singular PDE of mixed parabolichyperbolic type was computed by [10]. In [5], the index of PDAEs with the sequence of matrices method was proposed. It is an effective method for the finite systems except for the question of the unbounded operator in PDAEs. However, 
it generalizes the Kronecker index in a rather functional analytic manner. Other researchers also investigated the index structure of PDAEs systems in the area of coupling nonlinear PDAEs systems [7], mixed index [12], and index determination algorithm [30].

Despite the complexity analysis of index on PDAEs, research in the area of control problem for PDAEs is relatively scarce. This approach was first applied to design energy based controller of a coupled wave-heat equation systems in [31]. Stabilizing the coupled PDE-ODE systems with boundary control was considered in [21]. For the averaged model of the switched power converter system [20], by using Lyapunov function, a nonlinear saturating control law was designed. Based on the infinite-dimensional state space representation theory, [19] addressed the linear quadratic regulator control of the PDAEs. The optimal control problem is treated using Operator Riccati Equation (ORE) approach. The previous methods $[22,30]$ are thought to be derived from DAEs theory. Other optimal control problems [18, 23, 32] are considered with parabolic type PDAEs.

For all above coupled PDE-ODE systems, parabolic distributed PDEs systems, or hyperbolic systems, the time state variables matrices in those systems are reversible. Nevertheless, some singular system like parabolic-elliptic PDAEs cannot be direct application of the above theoretical study. The present work focuses on the development of a generalization stability method for a class of PDAEs with singular time derivative coefficient matrix. Our study derived from the article [13] in which there is a search for series solutions of PDAEs with method on DAEs. Moreover, motivated by the technique in $[17,33]$, our singular PDAEs system is considered in high-dimensional bounded domain.

The organization of this study is as follows. The problem statement for some singular time derivative PDAEs is given in Section 2. In Section 3, the original PDAEs are described as infinite-dimensional singular systems. And the state variables expression is built with Kronecker-Weierstrass form. Furthermore, the spectrum analysis is proposed to show the convergence property of the PDAEs solution. In Section 4, some dynamical stability property is analyzed with energy estimation method. Finally, in Section 5, as an application, we build some PDAEs model on some wetland conservation system with social behaviours. The applicability of the proposed approach is confirmed by the numerical simulation.

Notations. $\mathbb{R}, \mathbb{R}^{n}$, and $\mathbb{R}^{n \times m}$ are the set of real numbers, the $n$-dimensional Euclidean space, and the set of all real $n \times$ $m$ matrices, respectively. $\|\cdot\|$ denotes the Euclidean norm for vectors. For a vector $x(t) \in \mathbb{R}^{n},\|x(t)\|_{\infty}=: \sup \|x(t)\|$. $x(t) \in \mathscr{L}_{\infty}$ if $\|x(t)\|_{\infty}<\infty$. For a symmetric matrix $M, M>(<) 0$ means that it is positive (negative) definite. $I$ is the identity matrix. The superscript $T$ is used for the transpose. Matrices, if not explicitly stated, are assumed to have compatible dimensions. For the convenience, we define the following Hilbert space:

$$
\mathscr{H} \triangleq\left\{x: \Omega \times[0,+\infty) \longrightarrow \mathbb{R}^{n},\|x\|_{2}<\infty\right\},
$$

with inner product and $\mathscr{H}_{2}$-norm, respectively, defined by

$$
\begin{aligned}
\langle x, y\rangle & \triangleq \int_{\Omega} x^{T} y d \mathbf{z} \\
\|x\|_{2} & \triangleq\left\{\int_{\Omega}\|x\|^{2} d \mathbf{z}\right\}^{1 / 2} .
\end{aligned}
$$

\section{Description of PDAEs}

We consider the linear partial differential algebra equations (PDAEs) system in $d$-dimensional bounded spatial domain with a state space description of the form

$$
\begin{aligned}
& E \frac{\partial x}{\partial t}=D \Delta x+A x+B u, \\
& y(t)=\int_{\Omega} C x d \mathbf{z}+v,
\end{aligned}
$$

subject to the boundary conditions (BCs)

$$
p_{\iota} x_{\iota}(t, \mathbf{z})+q_{\iota} \frac{\partial x_{\iota}(t, \mathbf{z})}{\partial \mathbf{n}}=0, \quad \mathbf{z} \in \partial \Omega, \iota \in \mathcal{\vartheta}_{\mathrm{BC}}
$$

and the initial conditions (ICs)

$$
x_{\kappa}(0, \mathbf{z})=x_{\kappa}^{0}(\mathbf{z}), \quad \mathbf{z} \in \Omega, \kappa \in \mathcal{\vartheta}_{\mathrm{IC}},
$$

where $E, A, D \in \mathbb{R}^{n \times n}, D \geq 0, B \in \mathbb{R}^{n \times n_{u}}, x=$ $\left[x_{1}(t, \mathbf{z}) \cdots x_{n}(t, \mathbf{z})\right]^{T} \in \mathbb{R}^{n}$ is the vector of state variables, $\Omega \subset$ $\mathbb{R}^{d}$ is the spatial domain of the process, $\mathbf{z}=\left(z_{1}, \ldots, z_{d}\right) \in \Omega$ is the spatial coordinate, $t \geq 0$ is the time, $\Delta=\sum_{i=1}^{d} \partial_{z_{i}}^{2}$ is the Laplace operator, $u(t, \mathbf{z}) \in \mathbb{R}^{n_{u}}$ is the manipulated input vector function, $y(t) \in \mathbb{R}^{n_{y}}$ is the measure output, and $v(t)$ is the measurement disturbance. $p_{l}$ and $q_{l}$ are given constants, $\mathbf{n}$ is the outward normal vector of $x$ on $\partial \Omega, \vartheta_{\mathrm{BC}} \subseteq\{1, \ldots, n\}$, $\vartheta_{\text {IC }} \subseteq\{1, \ldots, n\}$, and $x_{\kappa}^{0}(\mathbf{z})$ is the spatial initial state function.

Remark 1. For system (3)-(6) we show that at least one of the matrices $E$ and $D$ is singular. The special case $D=0$ leads to DAEs. Therefore in this study we assume that $D$ is not a zero matrix. Another special case, $|E| \neq 0$, leads to the PDE-ODE systems which some researchers investigated [20]. In contrast to the problems with nonsingular matrices $E$ and $D$, the BCs (5) and ICs (6) have to meet certain consistency conditions. Thus for given PDAEs (3) the subsets $\vartheta_{\mathrm{BC}}$ and $\vartheta_{\text {IC }}$ are determined by consistency conditions (see [28]). In general, the BCs for $\iota \notin \vartheta_{\mathrm{BC}}$ and the ICs for $\kappa \notin \mathcal{\vartheta}_{\text {IC }}$ must be determined with the help of the PDAEs (3). With different $p_{t}$

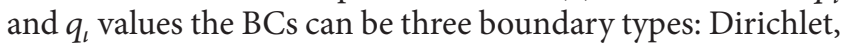
Neumann, and Robin boundary conditions. We assume that the BCs are homogeneous for simplicity and thought that our proposed method is applicable to the general inhomogeneous boundary conditions. Moreover, we assume that the process in (3)-(6) evolves on a compact set; that is, $(x(t, \mathbf{z}), u(t)) \epsilon$ $X \times U$ for all $t \geq 0$, where $X \times U \subset \mathbb{R}^{n} \times \mathbb{R}^{n_{u}}$ is a compact set containing the origin. 


\section{Decomposition of PDAEs and Spectrum Analysis}

3.1. Decomposition of PDAEs and Infinite Singular Systems. In this section, the PDAEs (3)-(6) will be rebuilt as a large infinite-dimensional system. Consider the linear elliptic operator

$$
\mathscr{D} x=D \Delta x+A x
$$

in $\Omega$ with homogeneous BCs (5). We denote by $\sigma(\mathscr{D})$ the spectrum of $\mathscr{D}$, that is, the set of $\lambda \in \mathbb{C}$ for which $(\mathscr{D}-\lambda I)$ is not invertible. More specifically, for the operator $\mathscr{D}$, the eigenvalue problem is defined as

$$
\begin{aligned}
\mathscr{D} \phi_{j}(\mathbf{z}) & =\lambda_{j} \phi_{j}(\mathbf{z}), \\
& (j=1, \ldots, \infty), \phi_{j}(\mathbf{z}) \in \mathscr{H}_{2, \Omega} \text { with BCs (5), }
\end{aligned}
$$

where $\lambda_{j} \in \mathbb{R}$ denotes the $j$ th eigenvalue and $\phi_{j}(\mathbf{z})$ denotes the corresponding orthogonal eigenfunction. Obviously, the eigenfunctions $\left\{\phi_{j}(\mathbf{z})\right\}_{j=1}^{\infty}$ form an orthogonal basis for eigenvalue problem (8). Furthermore, in the next subsection, we show that $\sigma(\mathscr{D})$ has discrete spectrum consisting only of real eigenvalues with at most a finite number of positive eigenvalues which is a generalization of [17]. Applying for PDE eigenvalue theory, we can obtain the following infinitedimensional differential algebraic equations (IDAEs) systems:

$$
\begin{aligned}
E \dot{X}_{j} & =\lambda_{j} X_{j}+B U_{j}, \quad j=1, \ldots, \infty \\
Y_{j} & =C_{j} X_{j}+V_{j},
\end{aligned}
$$

with the initial condition

$$
X_{j}(0)=\left\langle x^{0}(\mathbf{z}), \phi_{j}(\mathbf{z})\right\rangle,
$$

where

$$
\begin{aligned}
X_{j} & =\left\langle x, \phi_{j}\right\rangle, \\
U_{j} & =\left\langle u, \phi_{j}(\mathbf{z})\right\rangle, \\
V_{j} & =\left\langle v, \phi_{j}(\mathbf{z})\right\rangle, \\
C_{j} & =C \int_{\Omega} \phi_{j}(\mathbf{z}) d \mathbf{z} .
\end{aligned}
$$

Remark 2. One cannot guarantee that each system is solvable without considering the regularity of the matrix pencil set $\left\{\left(s E, \lambda_{j} I\right)\right\}_{j=1}^{\infty}$. Thus in this study we assume that for each $j$ the matrix pencil with respect to system (9) and (10) is regular. Furthermore, all systems have the uniform differential time index [13]; that is, for all $j$, the pencil $\left(s E, \lambda_{j} I\right)$ is regular and has the Riesz index $v_{d, j}=v_{d}$ (independent of $j$ ).

3.2. Eigenvalue Estimation and Property. For the unforced PDAEs

$$
E \frac{\partial x}{\partial t}=D \Delta x+A x
$$

with the corresponding IDAEs

$$
E \dot{X}_{j}=\lambda_{j} X_{j}, \quad j=1, \ldots, \infty .
$$

For every $j$, firstly, let us denote by $\sigma\left(E, \lambda_{j} I\right)$ the generalized eigenvalue set of systems (14), where $\lambda_{j}$ is defined by (8). We also write $\sigma(E)=\sigma(I, E)$ is the usual eigenvalue set. The subsequent theorem concerning the eigenvalues estimation of $\lambda_{j}$ for (14) corresponds to systems (3)-(6). Before that, we give two lemmas about the spectrum structural property of the scalar elliptic operator.

Lemma 3 (see [34]). With the strongly scalar elliptic operator $P(P$ is strongly elliptic in the sense that there exists $\gamma>0$ such that, for all $\left.\xi, \sum_{i, j} p_{i, j}(\mathbf{z}) \xi_{i} \xi_{j} \geq \gamma|\xi|^{2}\right)$ the operator defined by $\mathscr{P} x \equiv P x+a x \equiv \sum_{i, j=1}^{d} \partial_{z_{j}}\left(p_{i, j}(\mathbf{z}) \partial_{z_{i}} x\right)+a(\mathbf{z}) x$ in the bounded domain $\Omega \in \mathbb{R}^{d}$ with homogeneous boundary conditions of the form

$$
\alpha(\mathbf{z}) \frac{d x}{d \mathbf{n}}+\beta(\mathbf{z}) x=0 \quad \text { on } \partial \Omega
$$

has discrete spectrum consisting only of real eigenvalues. If a $(\mathbf{z})$ is bounded in $\Omega, \mathscr{P}$ can have at most a finite number of positive eigenvalues.

Lemma 4 (the Bauer-Fike Theorem). Assume that $A \in C^{n, n}$ is a diagonalizable matrix and $V \in C^{n, n}$ is the nonsingular eigenvector matrix such that $A=V \Lambda V^{-1}$, where $\Lambda=$ $\operatorname{diag}\left(\mu_{1}, \mu_{2}, \ldots, \mu_{n}\right)$ is a diagonal matrix. Let $\lambda$ be an eigenvalue of $A+T$; then there exists $\mu \in \sigma(A)$ such that $|\mu-\lambda| \leq$ $\|V\|_{2}\|T\|_{2}\left\|V^{-1}\right\|_{2}$.

Theorem 5. For the eigenvalue problem (8) the operator $\mathscr{D}$ has discrete spectrum consisting only of real eigenvalues. And there is a finite number of positive eigenvalues; that is, if all eigenvalues $\lambda_{j}$ are ordered such that $\lambda_{j}>\lambda_{j+1}$, then there exists a finite number $g$ so that $\lambda_{g+1}<0$ and $\varepsilon \triangleq\left|\lambda_{1}\right| /\left|\lambda_{g+1}\right|<1$ is a small positive number.

Proof. For every component $x_{k}(t, \mathbf{z})$ of state vector $x$, let $\mu_{j}^{k}$ denote the eigenvalue of the Sturm-Liouville (S-L) problem:

$$
\Delta x_{k}(t, \mathbf{z})+\mu_{j}^{k} x_{k}(t, \mathbf{z})=0 \quad \text { subject to }(5),
$$

where $\mu_{j}^{k}$ denotes the eigenvalue of (16). It is true that all $\mu_{j}^{k}$ satisfy Lemma 3 . And from the assumption that $D \geq 0$ one can get that, for every given $j$, if, for all $k=1, \ldots, n, \mu_{j}^{k}<0$, then all eigenvalue of $D \Delta x$ is negatively defined. Therefore, by Lemma $4,\left|\mu_{j}^{k}-\lambda_{j}\right| \leq c$, holds for every $j$ where $c$ is some given constant independent form $j$. Noticing that $\mu_{j}^{k} \rightarrow-\infty(j \rightarrow$ $\infty)$, the conclusion holds.

Remark 6. It should be pointed out that the existing onedimensional result [17] cannot be directly generalized to high-dimensional case because of the mathematical complexity property of the S-L problem on high-dimensional spatial space. Additionally, zero eigenvalue $\lambda_{j}=0$ yields the trivial 
constant state response $x(t, \mathbf{z})=$ const., which is outside the scope of interest of our study.

From Theorem 5 there exists some $p \in \mathbb{Z}^{+}$such that the operator $\mathscr{D}$ with respect to $(8)$ has discrete spectrum structure:

$$
\sigma(\mathscr{D})=\left\{\lambda_{1} \geq \lambda_{2} \geq \cdots \geq \lambda_{p} \geq \lambda_{p+1} \geq \cdots\right\}
$$

and $\lambda_{p+1}<0$. Now for the matrix pencil $\left(E, \lambda_{j} I\right)$ with respect to IDAEs (9), if $s_{j} \in \sigma\left(E, \lambda_{j} I\right)$ and $e \in \sigma(E)$, then from the definition of eigenvalue, the relationship among $\lambda_{j}, s_{j}$, and $e$ is $s_{j}=\lambda_{j} / e$. Immediately we have the following spectrum structure theorem.

Theorem 7. Assume $\left\{\lambda_{j}\right\}_{j=1}^{\infty}$ is the spectrum set of the eigenvalue problem (8) with respect to systems (9). $e \in \sigma(E)$ is the eigenvalue of $E$; then there exists some positive number $p>0$ such that, for every $j \in \mathbb{Z}^{+}, j>p$, the sign of the real part of $s_{j}$ is reversed with e. If the matrix pencil family set $\left\{\left(E, \lambda_{j} I\right)\right\}_{j=1}^{\infty}$ is admissible, then E must be a nonnegative matrix.

3.3. State and Output Response of PDAEs. In what follows we shall assume that each pencil $\left(E, \lambda_{j} I\right)$ with respect to (9) is regular and impulse-free. Intuitively, the KroneckerWeierstrass equivalent form of DAEs [1] can be applied to our systems. However, considering the nonnegative diffusion matrix $D$, this equivalent transformation leads to an unexpected matrix. And the spectrum analysis theorem above is no longer valid. Thus the Jordan transformation is introduced to solve the problem.

For the IDAEs (9), there exists nonsingular matrix $M$ such that $\left(E, \lambda_{j} I\right)$ can be transformed into the following form:

$$
M^{-1}\left[E, \lambda_{j} I\right] M=\left[\left(\begin{array}{cc}
E_{1} & 0 \\
0 & J
\end{array}\right),\left(\lambda_{j} I\right)\right],
$$

where $J \in \mathbb{R}^{n \times n}$ is a nilpotent matrix with index $n_{0}$ and $E_{1} \in$ $\mathbb{R}^{r \times r}$ is a nonsingular matrix. It follows immediately from the linear singular systems theory [6] that the state response of such system is

$$
X_{j}(t)=\left[X_{j, \text { reg }}(t), X_{j, \text { nil }}(t)\right]^{T},
$$

where $X_{j, \text { reg }}(t), X_{j, \text { nil }}(t)$ are given by

$$
\begin{aligned}
X_{j, \text { reg }}(t)= & M e^{\lambda_{j} E_{1}^{-1} t} X_{j, \text { reg }}(0) \\
& +M \int_{0}^{t} e^{\lambda_{j} E_{1}^{-1}(t-s)} B_{1} U_{j}(s) d s, \\
X_{j, \text { nil }}(t)= & -M \sum_{i=0}^{n_{0}-1} \lambda_{j}^{-(i+1)} J^{i} B_{2} U_{j}^{(i)}(t) .
\end{aligned}
$$

$B_{1}$ and $B_{2}$ are the components of $M B$ corresponding to the partition of the vector $X_{j}(t)$ into the regular and nilpotent parts. Thus the PDAEs (3)-(6) have the state response

$$
x(t, \mathbf{z})=\sum_{j=1}^{\infty} X_{j}(t) \phi_{j}(\mathbf{z})
$$

and the output response

$$
y(t)=\sum_{j=1}^{\infty}\left(C_{j} X_{j}(t)+V_{j}(t)\right),
$$

where $C_{j}, V_{j}(t)$ and $\left(j \in \mathbb{Z}^{+}\right)$are defined by (12).

The above state and output responses discussion provides a generalized systems theoretical approach to the PDAEs. From (20) we know that if there exists an eigenvalue of $E_{1}$ with negative real part then the solution grows exponentially which coincides with the "explosive solution" from a mathematical perspective. It should be noted that our results generalize the existing works $[19,21]$ in the aspect of derivative matrix $E$.

For the orthogonal property of the eigenfunction set $\left\{\phi_{j}\right\}_{j=1}^{\infty}$ some dynamical properties of the PDAEs including stability, stabilizability, and detectability can be given through a direct application of the LMI technique about the generalized systems theory $[6,35]$. The following theorem shows the admissibility of PDAEs via LMIs.

Theorem 8 (admissibility via LMIs). The PDAEs (13) are admissible if and only if there exists a matrix $P$ such that the first subsystem of IDAEs (14) is admissible; that is,

$$
\begin{aligned}
E^{T} P & =P^{T} E \geq 0 \\
\lambda_{1}\left(P^{T}+P\right)+P^{T} A+A^{T} P & <0
\end{aligned}
$$

where $\lambda_{1}$ is the first eigenvalue of the spatial differential operator (7).

Proof. Noticing that all eigenvalues $\lambda_{j}$ are ordered as $\lambda_{j} \geq$ $\lambda_{j+1}$, it can be easily deduced from the above that

$$
\lambda_{j}\left(P^{T}+P\right)+P^{T} A+A^{T} P<0
$$

holds for every $j \in \mathbb{Z}^{+}$. Then the desired result follows immediately by the admissible theory of the pencil $(E, A)$ (see [36] for details).

\section{Stability Analysis}

In view of Theorems 7 and 8 above, the systems' stability can be determined by spectrum analysis and LMIs. This validity of the above stability analysis relies on the convergence properties about the IDAEs. In the following, we propose some exponential stability property on the PDAEs by the energy estimation theory. For the following considerations, it will be simplest to assume homogeneous Neumann boundary conditions. 
Lemma 9 (see [34]). Let $x \in W_{2}^{2}(\Omega)$; then if $\mu_{1}$ is the smallest positive eigenvalue of $-\Delta$ on $\Omega$ (with the appropriate boundary conditions), the following Poincaré inequalities hold:

$$
\begin{aligned}
& \|\nabla x\|^{2} \geq \mu_{1}\|x-\bar{x}\|^{2}, \\
& \|\Delta x\|^{2} \geq \mu_{1}\|\nabla x\|^{2} \\
& \quad \text { if } \frac{d x}{d \mathbf{n}}=0 \text { on } \partial \Omega ; \\
& \|\nabla x\|^{2} \geq \mu_{1}\|x\|^{2} \quad \text { if } x=0 \text { on } \partial \Omega,
\end{aligned}
$$

where $\bar{x}=(1 /|\Omega|) \int_{\Omega} x d \mathbf{z}$.

Theorem 10. Assume that $x(t, \mathbf{z})$ is a bounded solution of (3) and (4) with homogeneous Neumann boundary conditions. Assume that $E \geq 0, D>0, \mu_{1}$ is the smallest positive eigenvalue of $-\Delta$ on $\Omega$, and $d_{1}$ is the smallest positive eigenvalue of $D$ and

$$
\delta=\frac{2\left(d_{1} \mu_{1}-\|A\|\right)}{\|E\|}>0 .
$$

Then

$$
\frac{1}{2} \int_{\Omega} \sum_{i=1}^{d} \frac{\partial x^{T}}{\partial z_{i}} E \frac{\partial x}{\partial z_{i}} d \mathbf{z} \leq c_{1} e^{-\delta t}
$$

for a positive constant $c_{1}$, and

$$
\int_{\Omega}\left\|x(t, \mathbf{z})-x_{M}(t)\right\| d \mathbf{z} \leq c_{2} e^{-\delta t}
$$

where $c_{2}$ is a positive constant and $x_{M}(t)=(1 /|\Omega|) \int_{\Omega} x(t, \mathbf{z}) d \mathbf{z}$ is the spatial average function; that is, the state variable vector $x(t, \mathbf{z})$ generated by the PDAEs is exponentially stable and asymptotically converges to its spatial average.

Proof. By introducing the energy integral (Lyapunov function),

$$
E_{L}(t)=\frac{1}{2} \int_{\Omega} \sum_{i=1}^{d} \frac{\partial x^{T}}{\partial z_{i}} E \frac{\partial x}{\partial z_{i}} d \mathbf{z}
$$

and compute

$$
\begin{aligned}
\frac{d}{d t} E_{L}(t) & =\int_{\Omega} \sum_{i=1}^{d} \frac{\partial x^{T}}{\partial z_{i}} E \frac{\partial^{2} x}{\partial z_{i} \partial t} d \mathbf{z} \\
& =\int_{\Omega} \sum_{i=1}^{d}\left(\frac{\partial x^{T}}{\partial z_{i}} \frac{\partial}{\partial z_{i}}(D \Delta x+A x)\right) d \mathbf{z} \\
& =\int_{\Omega} \sum_{i=1}^{d}\left(\frac{\partial x^{T}}{\partial z_{i}} D \frac{\partial \Delta x}{\partial z_{i}}+\frac{\partial x^{T}}{\partial z_{i}} A \frac{\partial x}{\partial z_{i}}\right) d \mathbf{z} .
\end{aligned}
$$

Noticing the Neumann BCs with the application of divergence theorem, we have

$$
\frac{d}{d t} E_{L}(t)=-\int_{\Omega} \Delta x^{T} D \Delta x d \mathbf{z}+\int_{\Omega} \sum_{i=1}^{d} \frac{\partial x^{T}}{\partial z_{i}} A \frac{\partial x}{\partial z_{i}} d \mathbf{z} .
$$

The above equality can be estimated as

$$
\frac{d}{d t} E_{L}(t) \leq-d_{1}\|\Delta x\|^{2}+\int_{\Omega} \sum_{i=1}^{d} \frac{\partial x^{T}}{\partial z_{i}} A \frac{\partial x}{\partial z_{i}} d \mathbf{z},
$$

where $d_{1}$ is the smallest positive eigenvalue of $D$.

According to Lemma 9, (33) implies that

$$
\frac{d}{d t} E_{L}(t) \leq-d_{1} \mu_{1}\|\nabla x\|^{2}+\|A\|\|\nabla x\|^{2}
$$

From the assumption that $-d_{1} \mu_{1}+\|A\|<0$, taking into account the fact that

$$
\begin{aligned}
E_{L}(t) & =\frac{1}{2} \int_{\Omega} \sum_{i=1}^{d} \frac{\partial x^{T}}{\partial z_{i}} E \frac{\partial x}{\partial z_{i}} d \mathbf{z} \leq \frac{\|E\|}{2} \int_{\Omega} \sum_{i=1}^{d} \frac{\partial x^{T}}{\partial z_{i}} \frac{\partial x}{\partial z_{i}} d \mathbf{z} \\
& =\frac{\|E\|}{2}\|\nabla x\|^{2},
\end{aligned}
$$

we obtain that

$$
\frac{d}{d t} E_{L}(t) \leq-\delta E_{L}(t)
$$

where

$$
\delta=\frac{2\left(d_{1} \mu_{1}-\|A\|\right)}{\|E\|}>0 .
$$

By Lemma 9, (36) implies (29).

Thus, under the conditions of the theorem, the time state response oscillations decay exponentially. The time-space state solution $x(t, \mathbf{z})$ asymptotically approaches the spatial average $x_{M}(t)$.

\section{Application to the Coastal Wetland Conservation System with Social Behaviour}

In this section, we illustrate through computer simulations the application of the theoretical development given above to some wetland conservation system with social behaviour on some plane rectangle domain (see Figure 1).

Our model derived from [37] in which a reactiondiffusion system incorporating one prey and two competing predator species under homogeneous Neumann boundary conditions was considered. In this model, human, bird (predators), and fish (prey) are chosen as the research objects. The spatiotemporal dynamics between bird and fish with 


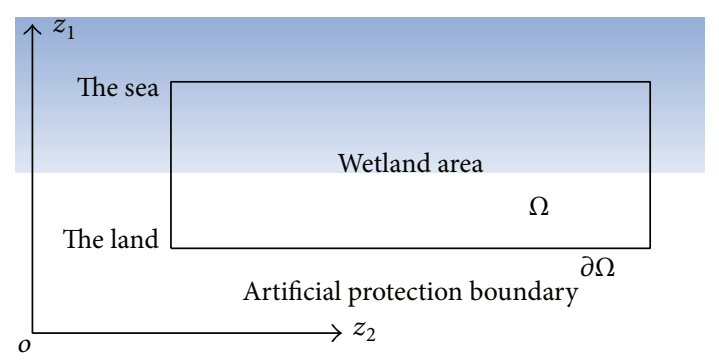

FIGURE 1: The idealized spatial domain is a rectangular domain with the sea oriented direction $z_{1}$ and coast line direction $z_{2}$. The wetland conservation is closed with no flux boundary conditions imposed.

human activity affected in a protected environment can be described by the following PDAEs:

$$
\begin{aligned}
\frac{\partial x_{1}}{\partial t}= & d_{1} \Delta x_{1}+r_{1} x_{1}\left(1-\frac{x_{1}}{N_{1}}-k_{1} \frac{x_{2}}{N_{2}}-h_{1} x_{3}\right), \\
\mathbf{z} \in \Omega, t>0, & \\
\frac{\partial x_{2}}{\partial t}= & d_{2} \Delta x_{2} \\
& +r_{2} x_{2}\left(-1+k_{2} \frac{x_{1}}{N_{1}}-\frac{x_{2}}{N_{2}}-h_{2} x_{3}\right), \\
0= & \Delta x_{3}+x_{3}, \quad \mathbf{z} \in \Omega, \quad \quad \mathbf{z} \in \Omega, t>0, \\
\frac{\partial x_{1}}{\partial \mathbf{n}}= & \frac{\partial x_{2}}{\partial \mathbf{n}}=\frac{\partial x_{3}}{\partial \mathbf{n}}=0, \quad \mathbf{z} \in \partial \Omega, t>0, \\
x_{1}(0, \mathbf{z})= & x_{1}^{0}(\mathbf{z}) \geq 0, \\
x_{2}(0, \mathbf{z})= & x_{2}^{0}(\mathbf{z}) \geq 0,
\end{aligned}
$$

where $x_{1}=x_{1}(t, \mathbf{z})$ and $x_{2}=x_{2}(t, \mathbf{z})$ represent the population of prey (birds) and predator (fish) species at time $t>0$ and spatial position $\mathbf{z} \in \Omega$, respectively; $x_{3}=x_{3}(\mathbf{z})$ represents the human population distribution; $d_{1}$ and $d_{2}$ stand for the spatial diffusion effects of fish and bird, respectively.

Fish population follows the logistic growth in the absence of bird with the intrinsic growth rate $r_{1}$ and the carrying capacity $N_{1} ; r_{2}$ is the death rate of bird with the carrying capacity $N_{2} ; k_{1}$ and $k_{2}$ represent the strength of relative effect of the interaction on the two species.

For a wetland ecosystem, the influence of human can be regarded as an invasive species and is not affected by other species. Thus the influence of human activities (e.g., the economic interest) on fish and bird is represented by $h_{1} x_{3}$ and $h_{2} x_{3}$, respectively (in the first two equations of system (38)). Human population in a state of free distribution can be described by

$$
\frac{\partial x_{3}(t, \mathbf{z})}{\partial t}=\Delta x_{3}(t, \mathbf{z})+x_{3}(t, \mathbf{z}), \quad \mathbf{z} \in \Omega, t>0
$$

Since the local human population distribution can reach a time independent dynamic balance in a short time, thus the above parabolic equation degenerates to the following elliptic equation:

$$
\Delta x_{3}+x_{3}=0, \quad \mathbf{z} \in \Omega
$$

Moreover, considering the geographical location effect of sea oriented direction we assume that human population $x_{3}\left(t, z_{1}, z_{2}\right)=x_{3}\left(t, z_{1}\right)$ is independent with the coast line direction. And the parameters $d_{i}, r_{i}, k_{i}$, and $h_{i}(i=1,2)$ are positive real numbers.

Systems (38) can be rewritten as the following PDAEs:

$$
E \frac{\partial x}{\partial t}=D \Delta x+f(x)
$$

where

$$
\begin{aligned}
E & =\left(\begin{array}{lll}
1 & 0 & 0 \\
0 & 1 & 0 \\
0 & 0 & 0
\end{array}\right), \\
x & =\left(x_{1}, x_{2}, x_{3}\right)^{T}, \\
D & =\operatorname{diag}\left(d_{1}, d_{2}, 1\right) \\
f(x) & =\left(\begin{array}{c}
r_{1} x_{1}\left(1-\frac{x_{1}}{N_{1}}-k_{1} \frac{x_{2}}{N_{2}}-h_{1} x_{3}\right) \\
r_{2} x_{2}\left(-1+k_{2} \frac{x_{1}}{N_{1}}-\frac{x_{2}}{N_{3}}-h_{2} x_{3}\right)
\end{array}\right) .
\end{aligned}
$$

It is obvious that the nonnegative equilibrium of system (38) is the solution of the following nonlinear equations:

$$
\begin{aligned}
& 1-\frac{x_{1}}{N_{1}}-k_{1} \frac{x_{2}}{N_{2}}-h_{1} x_{3}=0, \\
&-1+k_{2} \frac{x_{1}}{N_{1}}-\frac{x_{2}}{N_{2}}-h_{2} x_{3}=0, \\
& x_{3}=0 .
\end{aligned}
$$

The nonnegative equilibrium is

$$
\left(\frac{N_{1}\left(k_{1}+1\right)}{1+k_{1} k_{2}}, \frac{N_{2}\left(k_{2}-1\right)}{1+k_{1} k_{2}}, 0\right)
$$

where $k_{2}>1$.

Now we consider the local stability of the equilibrium. We choose $\Omega:=\left\{\left(z_{1}, z_{2}\right) \mid 0<z_{1}<\pi, 0<z_{2}<1\right\}$. To the consistency conditions the initial conditions are taken as $x_{1}^{0}(\mathbf{z})=0.3$ and $x_{2}^{0}(\mathbf{z})=0.3\left(1+\cos \left(z_{1}\right)\right)$. The remaining system parameters are chosen as $N_{1}=N_{2}=1, r_{1}=2$, $r_{2}=0.2, k_{1}=8, k_{2}=18$, and $D=\operatorname{diag}(2,3,1)$. By direct 


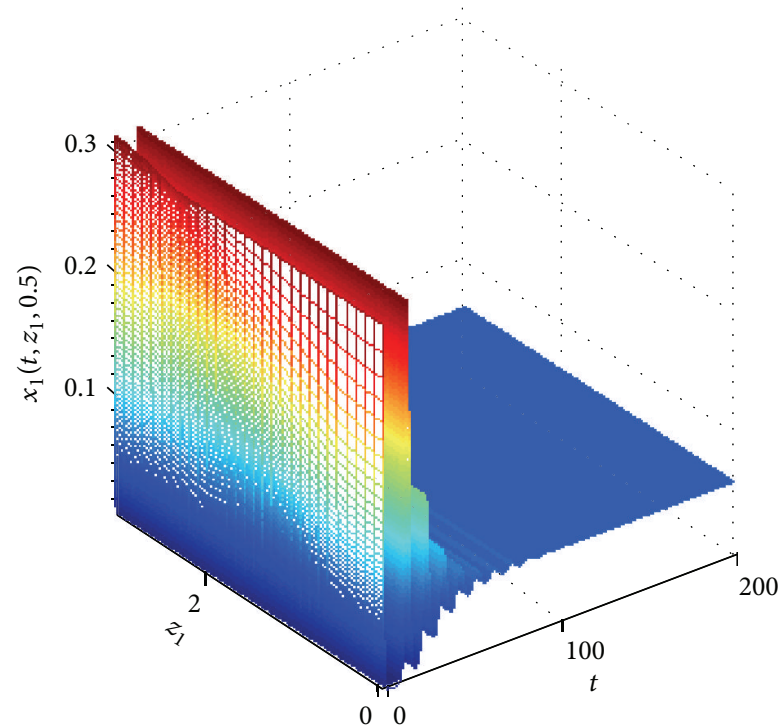

(a)

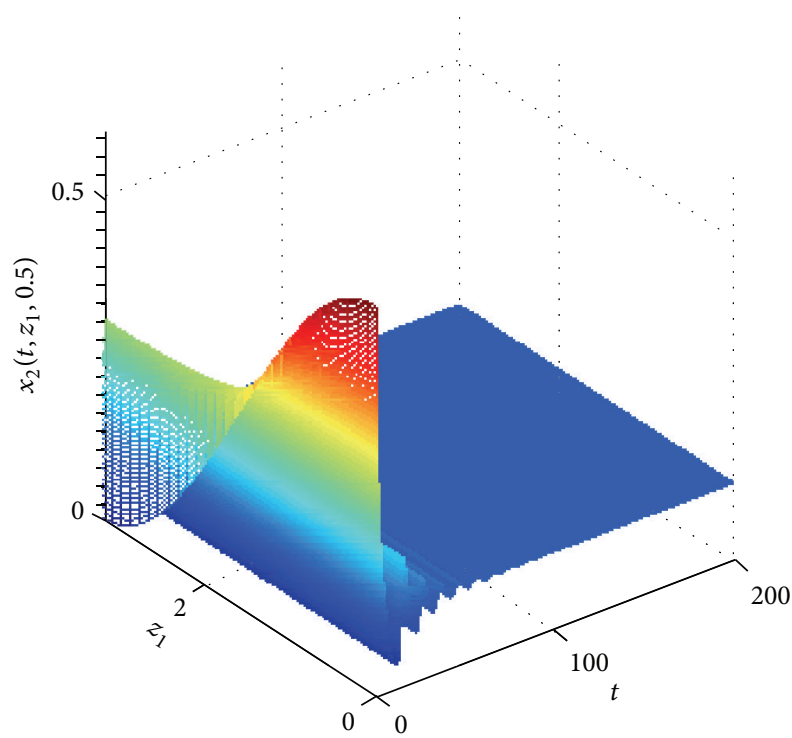

(b)

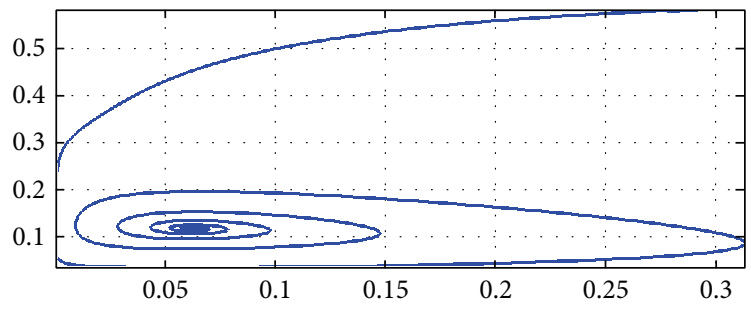

(c)

Figure 2: Numerical results for the state estimation analysis. (a) Time spatial response $x_{1}\left(t, z_{1}, 0.5\right)$ in $\left(t, z_{1}\right)$ domain. (b) Time spatial response $x_{2}\left(t, z_{1}, 0.5\right)$ in $\left(t, z_{1}\right)$ domain. (c) The phase trajectory about $x_{1}, x_{2}\left(h_{1}=0.2, h_{2}=0.1\right)$.

TABLE 1: Values of the simulation parameters.

\begin{tabular}{lcc}
\hline Case & $\left\|A_{J}\right\|$ & $d_{1} \mu_{1}-\left\|A_{J}\right\|$ \\
\hline$h_{1}=h_{2}=0.1$ & 1.0069 & 1.9931 \\
\hline$h_{1}=24, h_{2}=0.1$ & 3.2841 & -0.7159 \\
\hline
\end{tabular}

computing we have the Jacobi matrix of the linearized system at the equilibrium:

$$
A_{J}=\left(\begin{array}{ccc}
-\frac{18}{145} & -\frac{144}{145} & -\frac{18 h_{1}}{145} \\
\frac{306}{725} & -\frac{17}{725} & -\frac{17 h_{2}}{725} \\
0 & 0 & 1
\end{array}\right)
$$

According to the stability analysis of Theorem 10 in Section 4, we consider the local stability of these PDAEs at the equilibrium in different parameter values (see Table 1). Due to the particular choice of the system parameters $h_{1}$ and $h_{2}$ the state variables $x_{1}$ and $x_{2}$ show some different dynamical properties. By increasing the value of $h_{1}$, the value of $n d_{1} \mu_{1}-\|A\|$ can be changed from positive to negative.
Simulation results for system $(38)$ in $\left(t, z_{1}\right)$ domain at $z_{2}=0.5$ are depicted in Figures 2 and 3. In Figures 2(a) and 2 (b) the state variables $x_{1}, x_{2}$ spatial-temporal show the corresponding exponential stability. It shows great difference with the case in Figure 3. Figure 4 shows the different spatial convergence property of the system. Ecologically, the stable prey-predator relationship stands when the diffusion rates of prey and predator $\operatorname{diag}\left(d_{1}, d_{2}, 1\right)$ are very high with low human effect rates $h_{1}$ and $h_{2}$ which correspond to the experience.

\section{Conclusion}

In this study we have studied the problem of some singular time derivative PDAEs in high-dimensional domain. With the decomposition ideas derived from PDE theory we built the IDAEs for reconstruction of PDAEs. Some admissible results of the IDAEs (9) corresponding the PDAEs (3) are proposed. An exponentially stable result on the PDAEs is presented through the energy estimation about the state variables under the homogenous Neumann boundary conditions for the positive diffusion matrix. Finally, as an application, we built some wetland conservation model with 


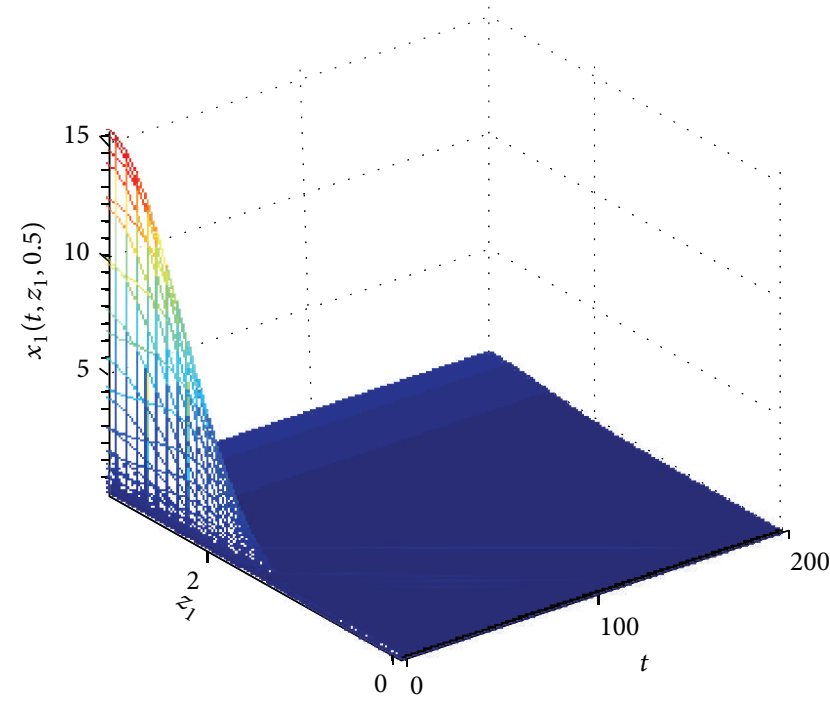

(a)

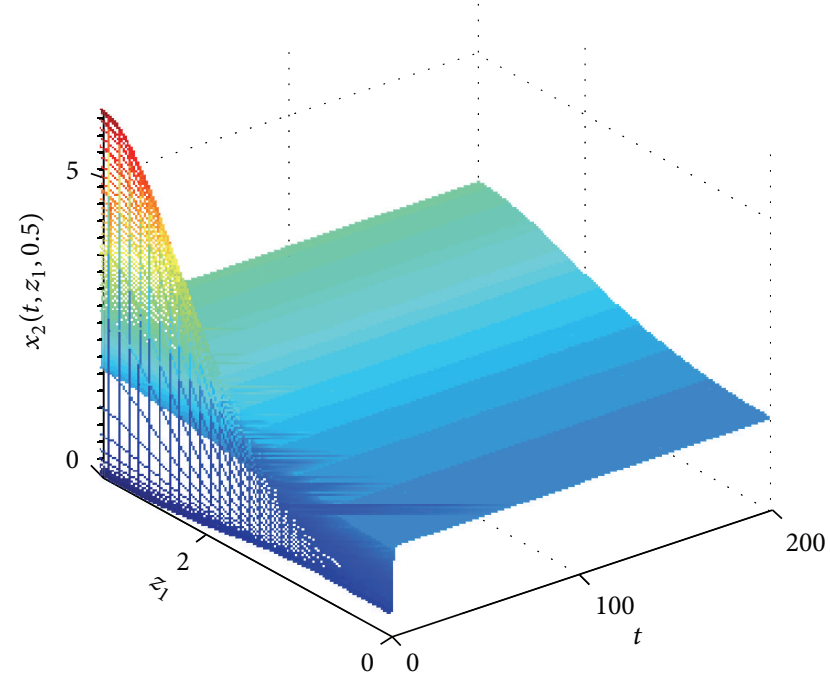

(b)

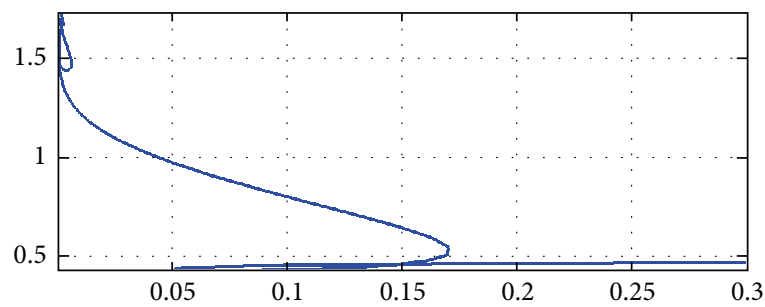

(c)

Figure 3: Numerical results for the state estimation analysis. (a) Time spatial response $x_{1}\left(t, z_{1}, 0.5\right)$ in $\left(t, z_{1}\right)$ domain. (b) Time spatial response $x_{2}\left(t, z_{1}, 0.5\right)$ in $\left(t, z_{1}\right)$ domain. (c) The phase trajectory about $x_{1}, x_{2}\left(h_{1}=24, h_{2}=0.1\right)$.
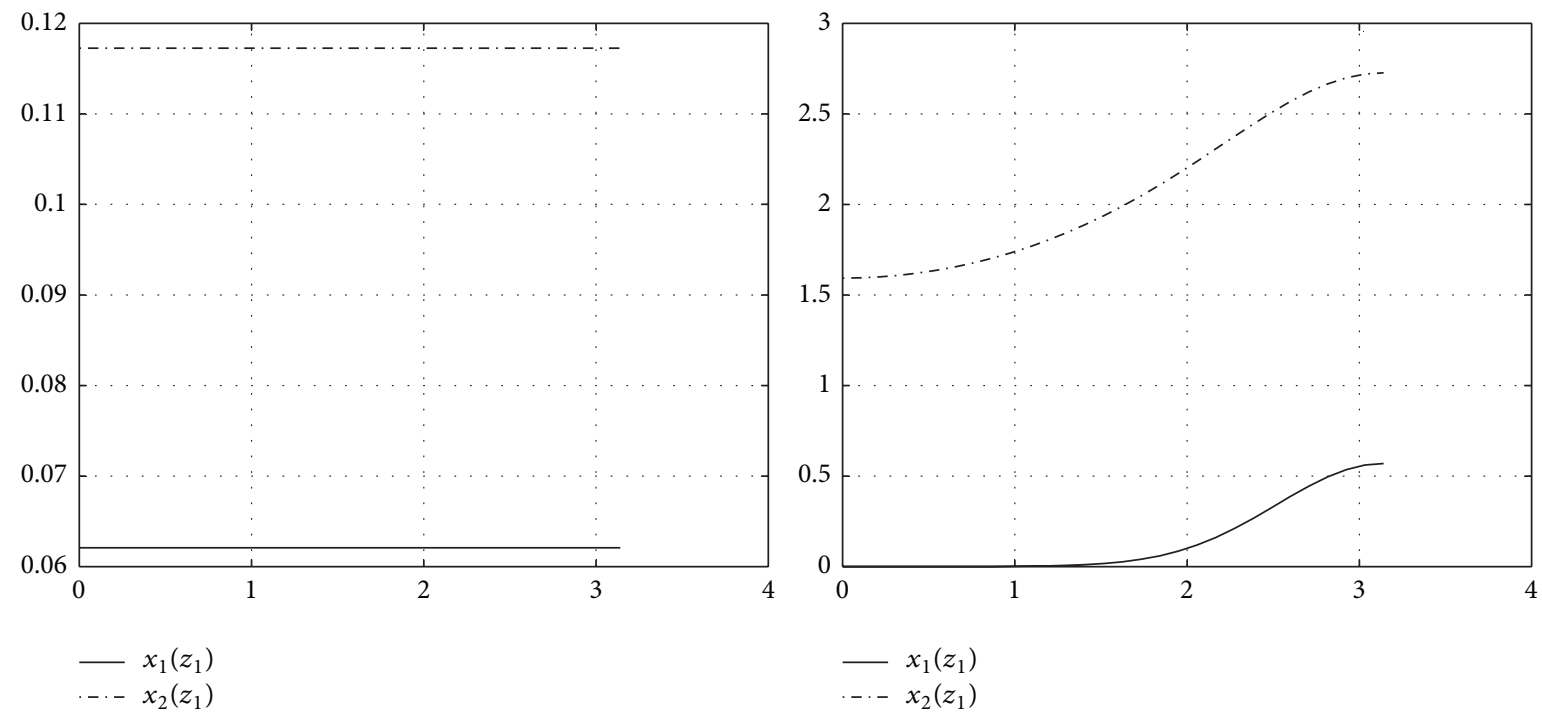

FIgURE 4: Numerical results on spatial convergence of $x_{1}, x_{2}$. 
social behaviour. The numerical results show the effectiveness of this development.

\section{Conflict of Interests}

The authors declare that they have no conflict of interests regarding the publication of this paper.

\section{Acknowledgments}

The research is supported by N.N.S.F. of China under Grant no. 61273008 and no. 61104003. The research is also supported by the Key Laboratory of Integrated Automation of Process Industry (Northeastern University). The authors are grateful to the anonymous referee for a careful checking of the details and for helpful comments that allow them to improve the paper.

\section{References}

[1] L. Dai, Singular Control Sysems, Springer, Berlin, Germany, 1989.

[2] S. L. Campbell, Singular Systems of Differential Equations II, Pitman, Wellington, New Zealand, 1982.

[3] F. L. Lewis, "A survey of linear singular systems," Circuits, Systems, and Signal Processing, vol. 5, no. 1, pp. 3-36, 1986.

[4] Q. Zhang, Decentralized Control and Robust Control for Singular Systems, Northwestern Polytechnical University Press, Xi'an, China, 1997.

[5] R. Riaza, Diferential-Algebraic Systems: Analytical Aspects and Circuit Applications, World Scientific Publishing, Singapore, 2007.

[6] Q. Zhang, C. Liu, and X. Zhang, Analysis and Control of Singular Biological Systems, vol. 421 of Lecture Notes in Control and Information Sciences, Springer, London, UK, 2012.

[7] G. Alì and N. Rotundo, "On the tractability index of a class of partial differential-algebraic equations," Acta Applicandae Mathematicae, vol. 122, no. 1, supplement I, pp. 3-17, 2012.

[8] G. Alì and N. Rotundo, "An existence result for elliptic partial differential-algebraic equations arising in semiconductor modeling," Nonlinear Analysis, Theory, Methods \& Applications, vol. 72, no. 12, pp. 4666-4681, 2010.

[9] S. L. Campbell and W. Marszalek, "The index of an infinitedimensional implicit system," Mathematical and Computer Modelling of Dynamical Systems, vol. 5, no. 1, pp. 18-42, 1999.

[10] J. Rang, L. Angermann, and J. Rang, "Perturbation index of linear partial differential-algebraic equations," Applied Numerical Mathematics, vol. 53, no. 2, pp. 437-456, 2005, Tenth Seminar on Numerical Solution of Differential and Differntial-Algebraic Euqations (NUMDIFF-10).

[11] K. Chudej, P. Heidebrecht, V. Petzet et al., "Index analysis and numerical solution of a large scale nonlinear PDAE system describing the dynamical behaviour of Molten Carbonate Fuel Cells," ZAMM-Zeitschrift für Angewandte Mathematik und Mechanik, vol. 85, no. 2, pp. 132-140, 2005.

[12] L. Jansen, "A semi-explicit formulation of a coupled electromagnetic field/circuit problem," Journal of Computational and Applied Mathematics, vol. 262, pp. 150-160, 2014.

[13] W. Lucht, "Partial differential-algebraic systems of second order with symmetric convection," Applied Numerical Mathematics, vol. 53, no. 2-4, pp. 357-371, 2005.
[14] K. Debrabant and K. Strehmel, "Convergence of Runge-Kutta methods applied to linear partial differential-algebraic equations," Applied Numerical Mathematics, vol. 53, no. 2-4, pp. 213229, 2005.

[15] J. Vuong and B. Simeon, "On finite element method-flux corrected transport stabilization for advection-diffusion problems in a partial differential-algebraic framework," Journal of Computational and Applied Mathematics, vol. 262, pp. 115-126, 2014.

[16] A. Bartel, M. Brunk, and S. Schöps, "On the convergence rate of dynamic iteration for coupled problems with multiple subsystems," Journal of Computational and Applied Mathematics, vol. 262, pp. 14-24, 2014.

[17] H.-N. Wu and H.-X. Li, "Robust adaptive neural observer design for a class of nonlinear parabolic PDE systems," Journal of Process Control, vol. 21, no. 8, pp. 1172-1182, 2011.

[18] B. Luo and H.-N. Wu, "Approximate optimal control design for nonlinear one-dimensional parabolic PDE systems using empirical eigenfunctions and neural network," IEEE Transactions on Systems, Man, and Cybernetics, Part B: Cybernetics, vol. 42, no. 6, pp. 1538-1549, 2012.

[19] A. A. Moghadam, I. Aksikas, S. Dubljevic, and J. F. Forbes, "Boundary optimal (LQ) control of coupled hyperbolic PDEs and ODEs," Automatica, vol. 49, no. 2, pp. 526-533, 2013.

[20] J. Daafouz, M. Tucsnak, and J. Valein, "Nonlinear control of a coupled PDE/ODE system modeling a switched power converter with a transmission line," Systems and Control Letters, vol. 70, pp. 92-99, 2014.

[21] S. Tang and C. Xie, "State and output feedback boundary control for a coupled PDE-ODE system," Systems \& Control Letters, vol. 60 , no. 8, pp. 540-545, 2011.

[22] D. Clever and J. Lang, "Optimal control of radiative heat transfer in glass cooling with restrictions on the temperature gradient," Optimal Control Applications \& Methods, vol. 33, no. 2, pp. 157175, 2012.

[23] L. Jadachowski, T. Meurer, and A. Kugi, "Backstepping observers for linear PDEs on higher-dimensional spatial domains," Automatica, vol. 51, pp. 85-97, 2015.

[24] S. L. Campbell and C. W. Gear, "The index of general nonlinear DAEs," Numerische Mathematik, vol. 72, no. 2, pp. 173-196, 1995.

[25] S. L. Campbell and W. Marszalek, "DAEs arising from traveling wave solutions of PDEs," Journal of Computational and Applied Mathematics, vol. 82, no. 1-2, pp. 41-58, 1997.

[26] W. Marszalek, Analysis of Partial Differential Algebraic Equations, Applied Mathematics, North Carlina State University, 1997.

[27] W. Marszalek and Z. Trzaska, "A boundary-value problem for linear PDAEs," International Journal of Applied Mathematics and Computer Science, vol. 12, no. 4, pp. 487-491, 2002.

[28] W. Lucht and K. Debrabant, "On quasi-linear PDAEs with convection: applications, indices, numerical solution," Applied Numerical Mathematics, vol. 42, no. 1-3, pp. 297-314, 2002.

[29] W. S. Martinson and P. I. Barton, "A differentiation index for partial differential-algebraic equations," SIAM Journal on Scientific Computing, vol. 21, no. 6, pp. 2295-2315, 2000.

[30] R. Lamour, R. März, and C. Tischendorf, Differential-Algebraic Equations: A Projector Based Analysis, Springer, Berlin, Germany, 2013.

[31] X. Zhang and E. Zuazua, "Polynomial decay and control of a 1- $d$ hyperbolic-parabolic coupled system hyperbolic-parabolic coupled system," Journal of Differential Equations, vol. 204, no. 2, pp. 380-438, 2004. 
[32] T. Reis and T. Selig, "Zero dynamics and root locus for a boundary controlled heat equation," Tech. Rep., University Hamburg, Hamburger Beitrige zur Angewandten Mathematik, Hamburg, Germany, 2014.

[33] R. Denk and T. Seger, " $L^{p}$-estimates for a transmission problem of mixed elliptic-parabolic type," Boundary Value Problems, vol. 2014, no. 1, article 22, 2014.

[34] J. Smoller, Shock Waves and Reaction-Diffusion Equations, Springer, Berlin, Germany, 1983.

[35] C. Yang, Q. Zhang, and L. Zhou, Stability Analysis and Design for Nonlinear Singular Systems, Springer, Berlin, Germany, 2012.

[36] S. Xun and J. James, Robust Control and Filtering of Singular System, Springer, Berlin, Germany, 2006.

[37] W. Ko and I. Ahn, "A diffusive one-prey and two-competingpredator system with a ratio-dependent functional response: I, long time behavior and stability of equilibria," Journal of Mathematical Analysis and Applications, vol. 397, no. 1, pp. 928, 2013. 


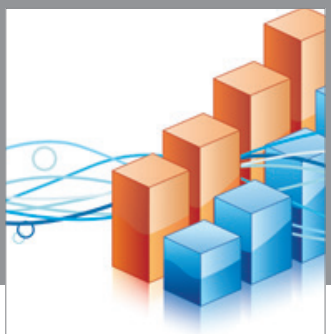

Advances in

Operations Research

vatem alat4

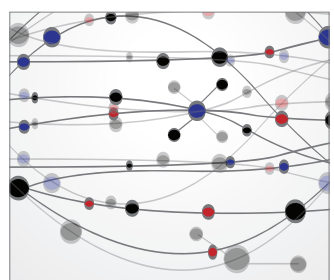

\section{The Scientific} World Journal
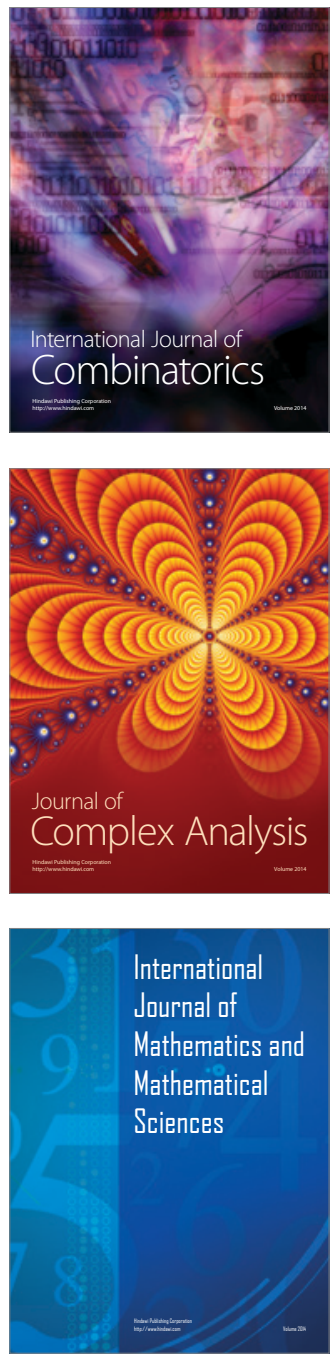
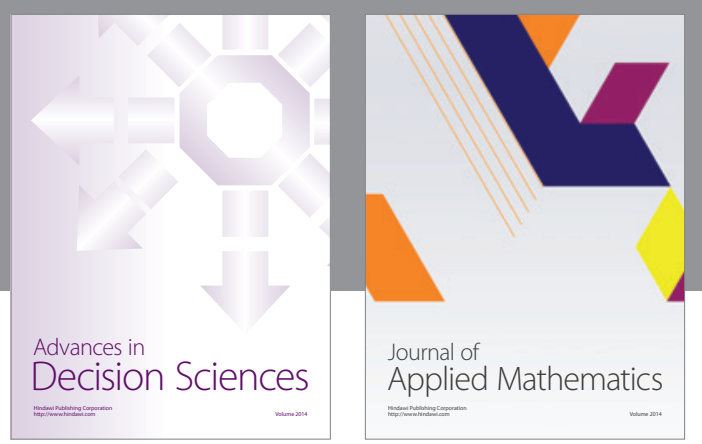

Algebra

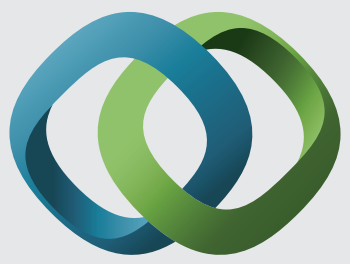

\section{Hindawi}

Submit your manuscripts at

http://www.hindawi.com
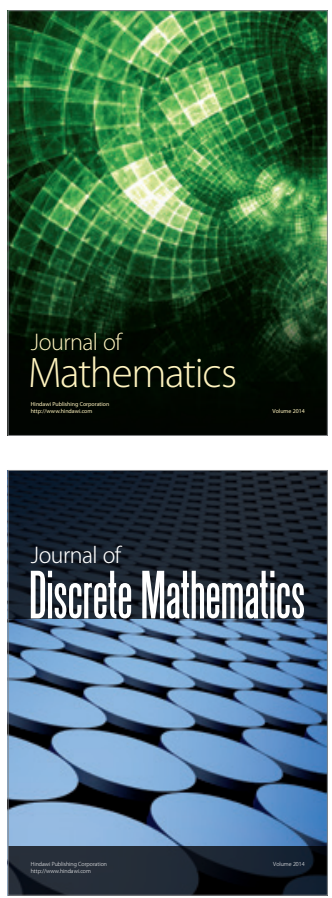

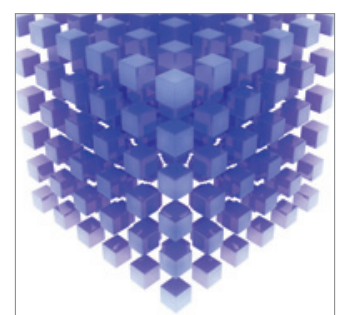

Mathematical Problems in Engineering
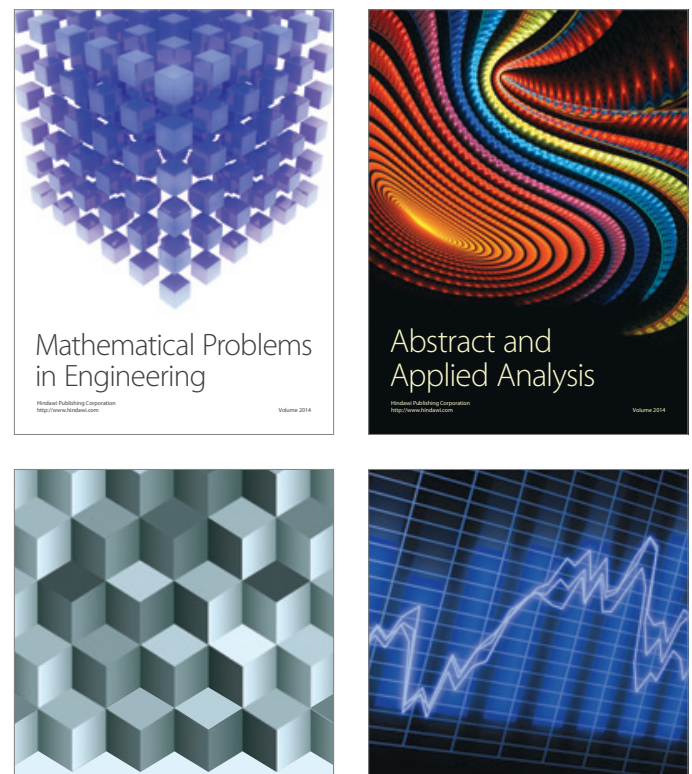

Journal of

Function Spaces

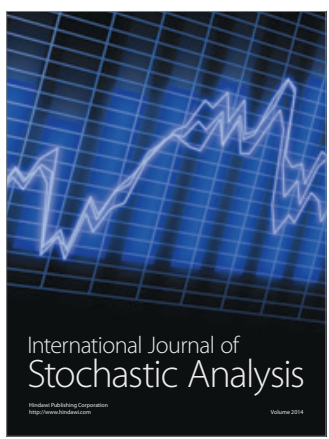

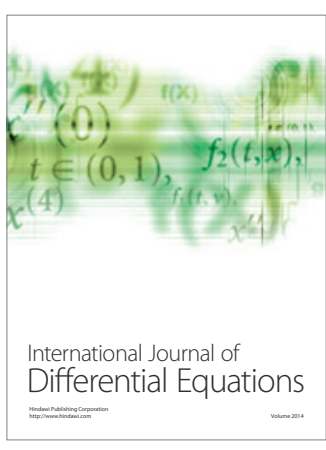
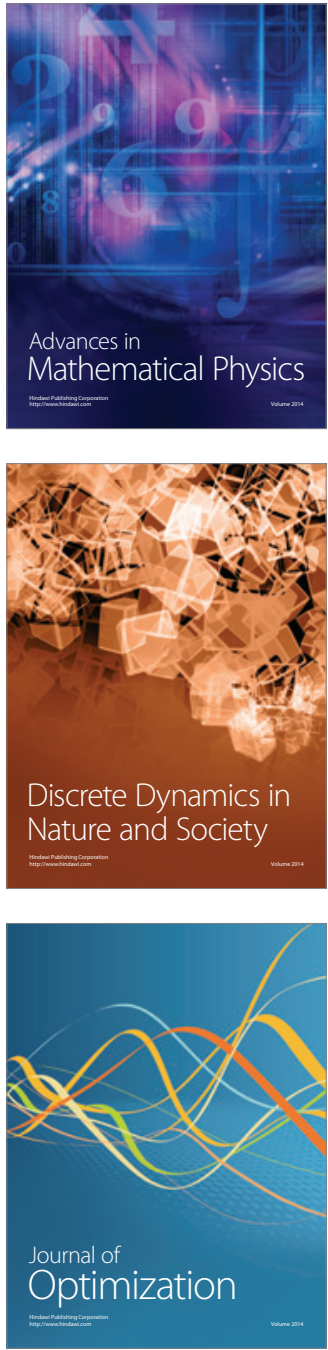